\title{
Childhood autoimmune hepatitis in a paediatric unit of a tertiary care hospital
}

\author{
Jia Ming $\underline{\text { Low }}^{1,2}$, MBBS, Michelle $\underline{\operatorname{Tan}}^{1,2}$, MBBS, MRCPCH, Agatha $\underline{\text { Garcia }}^{1,2}$, MD, DPPS, Marion ${\underline{A w^{1,2}}}^{1,2 B B S, ~ M M e d, ~}$ \\ Seng Hock Quak ${ }^{1,2}$, MBBS, MMed
}

INTRODUCTION Although childhood autoimmune hepatitis (AIH) has been extensively investigated in the West, data on AlH in the East is lacking. We aimed to investigate AlH's clinical, biochemical and histological features, as well as its outcomes, in one of Singapore's two major paediatric units.

METHODS This was a retrospective study of children diagnosed with AlH in the paediatric unit of National University Hospital, Singapore, over the last 12 years. Children with de novo AlH after liver transplantation were excluded. The demographic and clinical features of the patients, and their laboratory, treatment and clinical outcomes were reviewed. RESULTS This study comprised ten patients (six females, four males), with a median age of 5.1 (range 2.1-13.8) years at diagnosis. Five patients had inflammatory bowel disease (IBD). Seven patients had type $1 \mathrm{AlH}$, and three had autoimmune sclerosing cholangitis (ASC) and IBD; none had type $2 \mathrm{AlH}$. The median level of aspartate aminotransferase at diagnosis was 183 (range 45-2,649) U/L. Prednisolone $1 \mathrm{mg} / \mathrm{kg} /$ day was prescribed at diagnosis for eight patients. Two patients were lost to follow-up and were treated symptomatically when they re-presented with end-stage liver disease. Azathioprine or mycophenolate mofetil was prescribed after 3-7 months of treatment. Normalisation of aminotransferase levels took an average of 5.3 (range 1-39) months.

CONCLUSION AIH is a rare but important cause of liver pathology. Children in this region with elevated aminotransferases or unexplained hepatomegaly should be screened for $\mathrm{AlH}$.

Keywords: aminotransferases, childhood autoimmune hepatitis, hepatomegaly, immunosuppressant therapy, liver pathology

\section{INTRODUCTION}

Autoimmune hepatitis $(\mathrm{AIH})$ is a rare progressive inflammatory liver disease. The prevalence of $\mathrm{AlH}$ in Singapore was reported to be 4 cases per 100,000 children,(1) while the worldwide prevalence was reported to be $2-17$ per 100,000 children. ${ }^{(2-5)}$ $\mathrm{AlH}$ results in the progressive destruction of hepatic parenchyma, which may subsequently develop into cirrhosis and hepatic failure if treatment is not initiated at an early stage.

Even though $\mathrm{AlH}$ has been extensively investigated in the West, data regarding the disease in Eastern regions is lacking. Furthermore, there is marked variability in the clinical manifestations of $\mathrm{AlH} .{ }^{(6,7)}$ Asymptomatic patients may be identified when they undergo routine health screenings, such as those required for school. In such a setting, elevated aminotransferase may be the only indicator of liver disease. At the other end of the spectrum, patients may present with acute liver failure. Most of the patients who present with acute liver failure have established cirrhosis on liver biopsy, suggesting that they had subclinical disease for some time prior to clinical presentation. In addition, some patients may also present with a variety of mild to severe, nonspecific symptoms such as fatigue, lethargy, nausea and abdominal pain.

Since Waldenstrom's first description of $\mathrm{AIH}$ as a form of chronic hepatitis in young women in 1950, ${ }^{(8)}$ the pathogenesis and clinical evolution of $\mathrm{AlH}$, as well as the efficacy of various therapeutic regimes, have been well studied in the West, making it a relatively better known entity there. ${ }^{(9,10)}$ In the East, however,
$\mathrm{AlH}$ is rare in the adult population and even more uncommon in the paediatric population. ${ }^{(11)}$ To date, only two cases (one from Malaysia and the other from Brunei) have been reported in the paediatric population of the whole Southeast Asia region. ${ }^{(12,13)}$ Hence, medical practitioners caring for children in this region may not be familiar with AlH.

In the present case series, we assessed the clinical, biochemical and histological features of $\mathrm{AlH}$, as well as the treatment and clinical outcomes of these children.

\section{METHODS}

This retrospective cohort study reviewed the case records of children who were diagnosed with $\mathrm{AlH}$ or autoimmune sclerosing cholangitis (ASC) between January 2000 and June 2012 in the Department of Paediatrics, National University Hospital, a tertiary care hospital in Singapore. These patients were referred to our hospital by local and regional general paediatricians, and general practitioners for either: (a) a workup of abnormal liver function tests; or (b) management of acute liver failure. Approval from the appropriate National Healthcare Group Domain Specific Review Board was obtained.

Both patients with $\mathrm{AIH}$ and those with ASC were included in the present case series, as the two disorders have overlapping features (e.g. liver damage due to an underlying autoimmune cause), with the latter condition characterised by bile duct damage and interface hepatitis. These patients were diagnosed with either AIH or ASC in the presence of underlying clinical

${ }^{1}$ Khoo Teck Puat-National University Children's Medical Institute, National University Health System, ${ }^{2}$ Department of Paediatrics, Yong Loo Lin School of Medicine, National University of Singapore

Correspondence: Dr Tan Li Nien Michelle, Associate Consultant, Department of Paediatrics, National University Health System, 1E Kent Ridge Road, NUHS Tower Block Level 12 Singapore 119228. michelle_In_tan@nuhs.edu.sg 
Table I. Demographics and clinical characteristics of the patients $(n=10)$

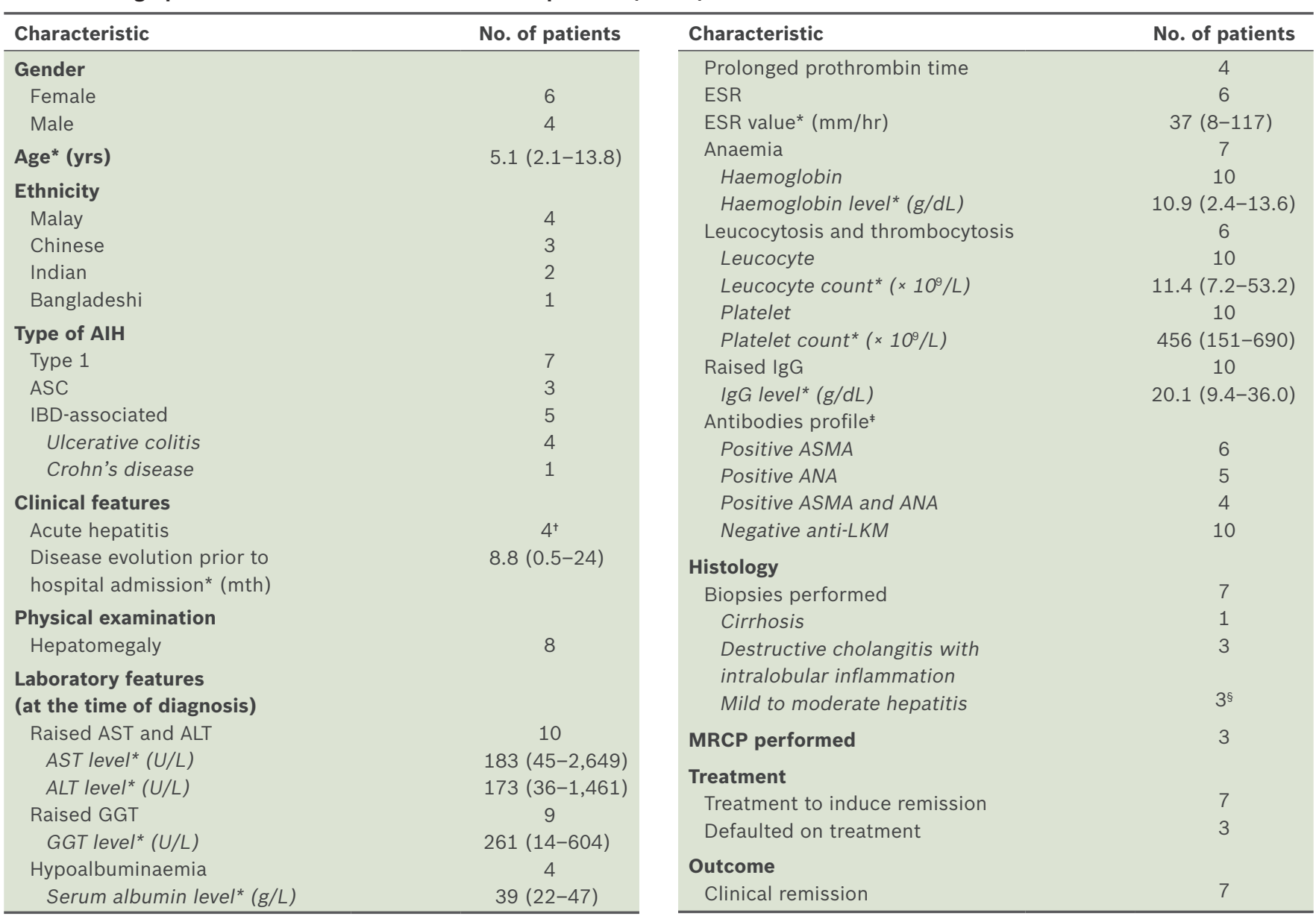

*Data is presented as median (range). tOne of the four patients defaulted on treatment and presented 40 months later with end-stage liver disease. $¥$ Three patients were negative for conventional antibodies (i.e. ANA, ASMA and anti-LKM, but had elevated IgG titres). ${ }^{8}$ Of the three patients, one had multinucleated giant cells and portal lymphoplasmacytic infiltrates with positive ASMA antibodies. AIH: autoimmune hepatitis; ALKM: anti-liver kidney microsomal; ALT: alanine transferase; ANA: antinuclear antibodies; ASC: autoimmune sclerosing cholangitis; ASMA: anti-smooth muscle antibodies; AST: aspartate aminotransferase; GGT: gamma-glutamyl transpeptidase; IBD: inflammatory bowel disease; ESR: erythrocyte sedimentation rate; IgG: immunoglobulin G; MRCP: magnetic resonance cholangiopancreatography

conditions (e.g. inflammatory bowel disease [IBD] and autoimmune diseases), biochemical evidence of liver disease, and/or serological features of autoimmunity in the absence of other known causes. We excluded patients who tested positive for (a) hepatitis A, B or C; (b) Epstein-Barr virus; (c) Cytomegalovirus; (d) Wilson's disease or other genetically based liver diseases; and (e) other potentially hepatotoxic conditions. Children with de novo AlH after liver transplantation were also excluded.

The variables analysed in the present study were age, gender, other associated autoimmune diseases, clinical presentation at diagnosis, biochemical parameters of liver function (i.e. aminotransferases, bilirubin, gamma-glutamyl transpeptidase, alkaline phosphatase, prothrombin activity and albumin), serum immunoglobulin levels, autoimmunity markers, histology, treatment, need for liver transplant and clinical outcome. The clinical presentations of the patients were analysed and grouped following confirmation of the diagnosis. The categories of clinical presentation were: (a) incidental finding; (b) acute (i.e. having symptoms similar to that of acute viral hepatitis); (c) chronic (i.e. ascites, hepatomegaly, splenomegaly and jaundice were observed); and (d) fulminant hepatitis. The patients were then classified according to whether they tested positive for type 1 antibodies (i.e. antinuclear antibodies and/or anti-smooth muscle antibodies [ASMAs]) and type 2 antibodies (i.e. anti-liver kidney microsomal [anti-LKM]). The liver biopsy results of the patients were also analysed. Repeat biopsies were not routinely performed among the patients in the present study.

In the present study, remission was defined as an improvement in aminotransferase and immunoglobulin G (IgG) levels, with negative or extremely low titre autoantibodies, and relapse was defined as an increase in serum aminotransferase levels after remission has been achieved.

\section{RESULTS}

A total of ten patients were diagnosed with AlH or ASC in our hospital over a period of 12 years. The majority of these patients was female $(n=6)$. Of the ten patients, five had IBD. The most common finding on physical examination at presentation was hepatomegaly (observed in eight patients). Five of these eight patients who presented with hepatomegaly had a concomitant enlarged spleen. Complement assays with complements C3 and C4 were performed for four patients, in whom systemic lupus was suspected; low complement levels were noted in only one patient. The clinical and biochemical characteristics of the patients are presented in Table I. 
In the present study, seven patients had biopsies done at diagnosis. No biopsy was done for the other three patients, as they had either acute liver failure $(n=1)$ or decompensated endstage liver disease $(n=2)$. Biopsies are not typically routinely done for patients with these conditions, as in such cases biopsies would not be able to provide additional information for diagnosis (e.g. usually hepatic necrosis would be seen on biopsies of patients with acute liver failure).

Magnetic resonance cholangiopancreatography (MRCP) was performed on three patients at presentation. Of these three patients, two showed features suggestive of ASC - one had a generalised, beaded appearance of the extrahepatic and intrahepatic bile ducts, with areas of narrowing interspersed with areas of mild dilatations, and the other patient showed subtle contour irregularity in only the right intrahepatic biliary duct, which was suggestive of early beading. MRCP for the third patient did not reveal any biliary tree dilatation.

To induce remission, treatment with prednisolone $1 \mathrm{mg} / \mathrm{kg} / \mathrm{day}$ (maximum $60 \mathrm{mg}$ daily) was initiated in all ten patients. However, three patients defaulted on treatment. In the seven patients who underwent regular treatment, the prednisolone dose was gradually tapered off over a period of 2-8 weeks, while maintaining the minimum dose necessary to keep the aminotransferases (i.e. alanine transferase and aspartate aminotransferase) within the normal range. Azathioprine $(0.5-2.0 \mathrm{mg} / \mathrm{kg} /$ day) was used as a corticosteroid-sparing agent, and was added if there was suboptimal response to prednisolone after 6-8 weeks of treatment, to avoid progression to cirrhosis. In refractory cases, drugs such as mycophenolate mofetil were used. One patient who presented with mild hepatic inflammation was treated with prednisolone only; her liver function tests progressively returned to normal after three months. The three patients who defaulted on treatment and subsequently presented with end-stage liver disease were on symptomatic treatment only.

Clinical remission was observed in seven patients with clinical hepatitis - they had normalisation of their liver function parameters within an average of 5.3 (range 1-39) months. Five of the six patients with positive ASMA at the time of diagnosis had negative ASMA titres 22 (range 1-81) months later. One patient with ASC developed ulcerative colitis 20 months later, and another with type I AIH developed ulcerative colitis 60 months later.

As of 2012, all except one patient (excluding the three patients with end-stage liver disease/acute liver failure) required a minimal quantity of prednisolone and azathioprine/mycophenolate mofetil to maintain clinical and biochemical remission. One of the three patients with end-stage liver disease/acute liver failure received a cadaveric liver transplant, but died 23 months later from chronic allograft rejection. Although cirrhosis was present in three of the ten patients at diagnosis, mortality was observed to be low and a majority of the patients remained clinically stable with a good quality of life with the use of long-term treatment.

\section{DISCUSSION}

Although the number of patients in our case series is small, the results obtained appear to be similar to those described by other authors; ${ }^{(14,15)}$ there was a predominance of female patients and heterogeneity of clinical presentation (ranging from incidental findings and cases masquerading as acute viral-like hepatitis to cases of end-stage liver disease).

As observed in other case series, the presence of other autoimmune conditions, such as ulcerative colitis, vitiligo, diabetes mellitus and vasculitis, can be associated with AIH/ASC..$^{(16,17)}$ In the present case series, five of our patients had IBD (four with ulcerative colitis and one with Crohn's disease). This proportion is relatively higher than that reported in Western studies. In Gregorio et al's study, only three of the 52 children had ulcerative colitis with AIH/ASC, ${ }^{(14)}$ and in a Norwegian case series by Olbjorn and Sanengen, only four cases of IBD (two with ulcerative colitis and two with Crohn's disease) were reported among their 18 patients with AIH/ASC. ${ }^{(15)}$ Hence, we suggest that clinical suspicion for AIH/ASC be raised in patients in our local population with autoimmune conditions and raised liver enzymes.

At present, none of the patients included in the present case series had had genetic studies performed, as genotyping is not required to render a diagnosis of AIH/ASC. However, consanguineous parentage was reported in one patient, lending some weight to the hypothesis that a combination of one or more genes, combined with environmental factors, may play a role in the aetiology of AlH/ASC. Although the genetic pattern of AIH/ASC has not been clearly established to date, an increased frequency of human leucocyte antigen- (HLA-) A1, B8 and DR3/DR4 have been observed in patients with type $1 \mathrm{AlH} .{ }^{(18)}$ In Caucasians, type 1 AlH was found to be strongly associated with HLA-DR3 and HLA-DR4 serotypes, whereas in the Japanese, it was found to be primarily associated with the HLA-DR4 serotype. ${ }^{(19)}$ Further studies need to be conducted to shed light on the pathogenesis of AIH/ASC, as its aetiology is currently unknown.

Children with AIH/ASC usually respond favourably to immunosuppressive treatment with corticosteroids, with or without azathioprine/mycofenolate mofetil. This was also observed in the present case series, where a majority of our patients required only a minimal quantity of immunosuppressants to maintain a state of stable remission. Nonetheless, children with AIH/ASC may develop liver cirrhosis and some may even require liver transplantation if the disease progresses. As seen in the three patients in our study who defaulted on follow-up, the progression to end-stage liver disease was more rapid than that of the patients who were on immunosuppressant therapy. Therefore, early diagnosis and improved treatment strategies are imperative for better prognosis for children with AIH/ASC.

The present case series was not without limitations. Firstly, as it was a retrospective study that involved the review of case records, it depended, to some extent, on the accuracy of the documentation of these case records. Secondly, even though it is the largest case series published to date on AIH/ASC in Southeast Asia, the number of cases is still relatively small. Given the relatively sparse prevalence of AIH/ASC and the small number of cases seen, we are unable to compare and contrast the indications and choice of treatment, which would include the reasons for the modalities chosen for the patients. However, it is precisely the 
fact that the prevalence of this disease is low that there is difficulty in obtaining a large sample size. Thus, the present study offers valuable insight into the demographic and clinical characteristics of patients with AIH/ASC in the East, enabling comparisons with patients from the West. We conclude from the results of the present study that the clinical characteristics of our patients are similar to that of Western patients.

Although $\mathrm{AlH}$ is a rare condition among paediatric patients in Eastern regions, it remains an important cause of liver disease in this age group. Physicians should consider this in the differential diagnosis, especially in asymptomatic children with raised aminotransferase levels, and those presenting with hepatic disease with an insidious course, and unexplained hepatomegaly or hepatosplenomegaly, when other conditions that can cause chronic hepatitis and diseases related to metabolic disorders have been excluded. This is important as the condition responds satisfactorily to immunosuppressant therapy, and prompt referrals to a tertiary care centre will allow for earlier treatment, and thus, better long-term prognosis.

\section{ACKNOWLEDGEMENTS}

We thank Dr Dimple Rajgor for her assistance in the preparation of the Domain Specific Review Board application, as well as for editing, formatting and submitting the manuscript for publication.

\section{REFERENCES}

1. Lee YM, Teo EK, Ng TM, Khor C, Fock KM. Autoimmune hepatitis in Singapore: a rare syndrome affecting middle-aged women. J Gastroenterol Hepatol 2001; 16:1384-9.

2. Boberg KM, Aadland E, Jahnsen J, et al. Incidence and prevalence of primary biliary cirrhosis, primary sclerosing cholangitis, and autoimmune hepatitis in a Norwegian population. Scand J Gastroenterol 1998; 33:99-103.

3. Boberg KM. Prevalence and epidemiology of autoimmune hepatitis. Clin Liver Dis 2002; 6:635-47.

4. Delgado JS, Vodonos A, Malnick S, et al. Autoimmune hepatitis in southern Israel: a 15-year multicenter study. J Dig Dis 2013; 14:611-8.

5. Deneau M, Jensen MK, Holmen J, et al. Primary sclerosing cholangitis, autoimmune hepatitis, and overlap in Utah children: epidemiology and natural history. Hepatology 2013; 58:1392-400.

6. Krawitt EL. Autoimmune hepatitis. N Engl J Med 2006; 354:54-66.

7. Roberts EA. Autoimmune hepatitis from the paediatric perspective. Liver Int $2011 ; 31: 1424-31$.

8. Waldenstrom J. Leber, Blutproteine und Nahrungseiweiss. Dtsch Ges Verdau Stoffwechselkr 1950; 15:113-119.

9. Roberts EA. Autoimmune hepatitis from the paediatric perspective. Liver Int 2011; 31:1424-31.

10. Mieli-Vergani G, Vergani D. Autoimmune liver diseases in children - what is different from adulthood? Best Pract Res Clin Gastroenterol 2011; 25:783-95.

11. Yeh $\mathrm{SH}, \mathrm{Ni} \mathrm{YH}$, Jeng $\mathrm{YM}$, et al. Emerging importance of autoimmune hepatitis in children in Taiwan, an endemic area for viral hepatitis. Pediatr Neonatol 2009; 50:65-9.

12. Lee WS, Saw CB, Sarji SA. Autoimmune hepatitis/primary sclerosing cholangitis overlap syndrome in a child: diagnostic usefulness of magnetic resonance cholangiopancreatography. J Paediatr Child Health 2005; 41:225-7.

13. Basir N, Yew TG, Telisinghe PU, Chong VH. Autoimmune hepatitis in children: a report of two cases. Singapore Med J 2011; 52:e27-30.

14. Gregorio GV, Portmann B, Reid F, et al. Autoimmune hepatitis in childhood: a 20-year experience. Hepatology 1997; 25:541-7.

15. Olbjørn C, Sanengen T. [Autoimmmune hepatitis in children]. Tidsskr Nor Laegeforen 2007; 127:19-22. Norwegian.

16. Li DY, Schwarz KB. Autoimmune hepatitis. Adolesc Med Clin 2004; $15: 131-43, x$

17. Czaja AJ. Autoimmune liver disease. Curr Opin Gastroenterol 2004; 20:231-40.

18. Czaja AJ, Manns MP. Advances in the diagnosis, pathogenesis, and management of autoimmune hepatitis. Gastroenterology 2010; 139:58-72.e4.

19. Yokosawa S, Yoshizawa K, Ota M, et al. A genomewide DNA microsatellite association study of Japanese patients with autoimmune hepatitis type 1. Hepatology 2007; 45:384-90. 